\title{
Modelos organizativos e isomorfismo institucional entre asociaciones de migrantes michoacanos en Los Ángeles, California
}

\author{
CARLOS ALBERTO GONZÁLEZ ZEPEDA* \\ LUIS ESCALA RABADÁN**
}

RESUMEN: En este artículo se examina la relación que existe entre los modelos organizativos que adoptan las asociaciones de migrantes mexicanos en Estados Unidos y la realización de obras y proyectos en materia de desarrollo local en las comunidades de origen en México. Para ello se utiliza como referente empírico el caso de los migrantes michoacanos establecidos en la zona metropolitana de Los Ángeles, California. Entre los factores que se analizan, destaca el proceso de isomorfismo institucional a través del cual las organizaciones buscan asemejarse a las demás al incorporar elementos organizativos legitimados que reducen la incertidumbre y las posibilidades de fracaso de las obras que llevan a cabo para promocionar el desarrollo local en sus lugares de origen a través del Programa $3 \times 1$ para Migrantes.

PALABRAS CLAVE: isomorfismo institucional, modelos organizativos, migración México-Estados Unidos, asociaciones de migrantes michoacanos, desarrollo local.

\footnotetext{
* Estudiante del programa de Doctorado en Ciencias Sociales y Humanidades en la Universidad Autónoma Metropolitana, Unidad Cuajimalpa, México.

**Docente-investigador y coordinador de la Maestría en Estudios Socioculturales de El Colegio de la Frontera Norte, México.
} 
ABSTRACT: In this article the relationship between organizational models that adopt the Mexican HTAs in the U.S. and the execution of works and projects in local development in the communities of origin in Mexico is examined. This is used as a benchmark for empirical Michoacán migrants established in the metropolitan area of Los Angeles, California. Among the factors discussed, the process of institutional isomorphism through which organizations seek to resemble other legitimate organizational elements that incorporate uncertainty and reduce the chances of failure of the works undertaken to promote local development in their places of origin through the $3 \times 1$ Program for Migrants is highlighted.

KEY WORDS: institutional isomorphism, organizational models, Mexico-U.S. migration, Michoacán migrant associations, local development. 


\section{INTRODUCCIÓN}

La mejor estructura no garantizará los resultados ni el rendimiento. Pero la estructura equivocada es una garantía de fracaso.

Peter Drucker

7 a migración internacional se ha convertido en un fenómeno de gran influencia en la realidad económica, política, social y cultural de los países receptores y emisores debido al impacto en las estructuras insti-

tucionales y el territorio. Este fenómeno no sólo transforma el espacio sino también el ámbito institucional al propiciar la configuración de una serie de ideas, valores e intereses de los migrantes en los lugares de origen y destino.

Diferentes perspectivas han tratado de explicar la movilidad de las personas y las actividades de los migrantes para vincular los lugares de origen y destino. Desde las «redes migratorias transnacionales» se plantea la migración expandiendo el territorio mediante lazos interpersonales transfronterizos que conectan a los migrantes y no migrantes a través de vínculos de parentesco, amistad, etnicidad o adscripción al lugar de origen (Levitt, 2001).

Los contactos generados por la consolidación de redes y la llegada cotidiana de migrantes a la comunidad de destino permiten la organización e intercambio de experiencias, prácticas y recursos en ambos lados de la frontera. A través de ellos es posible comprender los procesos que transforman el espacio social en el territorio. Entre más institucionalizadas lleguen a ser estas relaciones, habrá más probabilidades de adscripción transnacional y fortalecimiento de las relaciones entre comunidades de llegada y origen.

Dentro de las redes trasnacionales surgen diferentes modelos de organización que van adoptando y adaptando los migrantes a sus necesidades individuales y colectivas. Tal es el caso de las asociaciones o clubes de migrantes oriundos basadas en la localidad, el municipio, la región o el estado de origen (Escala Rabadán, 2005; García Zamora, 2005; Moctezuma, 2011). Estas formas o modelos de organización con frecuencia imitan diversos elementos estructurales de otras organizaciones, no sólo para competir por los recursos, sino también para competir por prestigio, poder político, legitimidad institucional y condición económica. 
El presente artículo analiza los modelos organizativos de estas asociaciones de migrantes en atención a la recaudación de fondos o remesas colectivas para el financiamiento de obras y proyectos con objeto de promover el desarrollo local de las comunidades de origen en México. El trabajo parte de la premisa de que existe una correlación entre los modelos organizativos adoptados por los clubes de migrantes y la realización de obras y proyectos eficientes financiados con dichas remesas colectivas en las comunidades de origen a partir de la apropiación de prácticas y rutinas organizativas de organizaciones aparentemente similares.

Si bien es cierto que el análisis de estas formas de organización ha despertado el interés académico desde distintas disciplinas, precisamente por las diversas actividades que durante décadas han venido realizando para promover el desarrollo y bienestar de las comunidades de origen en México, también es cierto que se ha examinado poco lo relativo a las prácticas, rutinas y procedimientos organizativos que se realizan en su interior. A través de los conceptos de isomorfismo institucional y modelos organizativos de la teoría organizacional trataremos de explicar las características cambiantes que subyacen en gran parte de la vida organizativa de los clubes de migrantes, al tiempo que transforman las habilidades de las membresías y la capacidad de acción de dichas asociaciones. Para ello utilizaremos la información de un proyecto de investigación, realizado entre 2011 y 2012, sobre 10 clubes de Michoacán establecidos en la Zona Metropolitana de Los Ángeles, California: Jiquilpan USA, Los Reyes, Paisano Cd. Hidalgo, Cherán, Los Ángeles La Palma, Social Atacheo, Pajacuarán, Parácuaro, Lombardía y Social Huetamo; tres federaciones de migrantes: la Federación de Clubes y Asociaciones de Michoacanos en Norteamérica (Fecademin), la Federación Californiana de Michoacanos (Fecademich) y la Federación Californiana Lázaro Cárdenas del Río. Si bien dicho conjunto no constituye una muestra aleatoria de las asociaciones de migrantes en Estados Unidos, consideramos que su comportamiento organizativo sí es lo suficientemente representativo de lo que ocurre entre dichas asociaciones en general.

$94 \frac{\text { PRIMER SEMESTRE } 2014}{\text { MIGRACIÓN Y DESARROLLO NÚM. } 22}$ 


\section{REDES MIGRATORIAS TRANSNACIONALES E ISOMORFISMO INSTITUCIONAL}

Las redes sociales de migración han surgido en un mundo cada vez más consciente de la importancia de los lazos transnacionales. Al estar basadas en la conexión de expectativas de reciprocidad y confianza se invocan como una explicación ejemplar de cómo los migrantes manejan sus relaciones en ambos lados de la frontera (Bashi, 2007; Levitt y Glick Schiller, 2004; Global Forum on Migration and Development, 2013).

Las redes sociales basadas en la adscripción compartida al lugar de origen se refieren al sentido de pertenencia de los migrantes a una misma comunidad de origen, y son las que nos sirven para explicar lo que estamos abordando en este trabajo: la influencia que éstas tienen para propiciar formas de interacción y asociación entre paisanos que promueven la cohesión y son un facilitador para reintegrar a las comunidades establecidas en ambos lados de la frontera, incluso si se presenta una situación de retorno (Levitt, 2011; Bashi, 2007; Massey et al., 1990).

Este tipo de redes creadas en el circuito migratorio «pueden consistir en vínculos fuertes o débiles, que conectan a las personas que tienen relaciones transnacionales con aquellos que no las poseen, pero que reciben influencias directas de los flujos de ideas, objetos y remesas colectivas dentro de su campo de relaciones sociales» (Levitt y Glick Schiller, 2004: 67). Uno de los ejemplos más comunes de estos vínculos, en una variante más formalizada, son los denominados clubes de migrantes oriundos que, entre otras actividades, se distinguen por promover proyectos de desarrollo en las comunidades de origen (Goldring, 2002; Bada, 2003; Moctezuma, 2011; Duquette-Rury, 2011).

Los clubes de migrantes: solidez organizacional

Uno de los fenómenos que ocurren dentro de las redes sociales basadas en el paisanaje es el surgimiento de vínculos sociales más directos y activos que 
permiten a los migrantes cultivar sus identidades y fortalecer la relación con las comunidades de origen, como ocurre en el caso de los clubes de oriundos. Buena parte de los trabajos sobre el tema subrayan que se trata de organizaciones conformadas por migrantes de primera generación, que logran establecer vínculos transnacionales cargados de dispositivos simbólicos y un sentido de identidad y pertenencia que permite fortalecer los lazos de paisanaje entre los migrantes y las comunidades oriundas (Levitt, 2001; Orozco y Welle, 2005; Moya, 2005; Escala Rabadán, Rivera-Salgado y Rodríguez, 2011). Sin embargo, este trabajo va más allá, al apuntar que dichas organizaciones tienen al menos un objetivo claro, la supervivencia, y aquellos miembros que no se interesen en conservarla, normalmente la abandonarán. Asimismo, el bienestar y legitimidad de la organización produce un interés colectivo en preservarla, ya que confiere prestigio a la membresía y conlleva mayores beneficios. ${ }^{1}$

Desde diversas perspectivas, que van desde la sociología, la economía e incluso la antropología, se ha considerado que estas asociaciones son una forma concreta de representar las redes sociales que sirven a los migrantes como mecanismo de solidaridad, ayuda y comunicación entre origen y destino (García Zamora, 2012; Moctezuma, 2011; Duquette-Rury, 2011). La consolidación de estas formas organizativas también tiene mucho que ver con el esfuerzo de organización que promueven los migrantes más antiguos en el lugar de destino, pues tienen el tiempo, los recursos y la voluntad para movilizar a sus paisanos, en contraste con los migrantes más recientes (González Gutiérrez, 1995).

En este sentido, y para el caso de los migrantes mexicanos en Estados Unidos, desde mediados de la década de los noventa del siglo xx, el incremento en el número de organizaciones conformadas por migrantes mexicanos fue significativo: en el periodo 1998-2003 pasaron de 441 clubes a 623

${ }^{1}$ Como lo han señalado los teóricos organizacionales, «la supervivencia organizacional recibe atención como objetivo porque hay que luchar por ella de forma ininterrumpida y nunca es automática [...] Desde este punto de vista, una organización es viable y sobrevive sólo en tanto el equilibrio de alicientes y contribuciones es positivo [...] para producir contribuciones voluntarias de participación y esfuerzo para mantener a la organización» (Pfeffer, 1997: 8).

$96 \frac{\text { PRIMER SEMESTRE } 2014}{\text { MIGRACIÓN Y DESARROLLO NÚM. } 22}$ 
respectivamente; mientras que entre 2009-2011 pasaron de 1,392 organizaciones a 1,842, en su mayoría organizaciones tipo clubes de oriundos (Rivera-Salgado, Bada y Escala Rabadán, 2005; SRE, 2009 y 2011). Al respecto, los estados mexicanos con mayor número de organizaciones de este tipo en Estados Unidos son Guanajuato con 450 organizaciones; Hidalgo, 134; Michoacán, 122; Jalisco, 93; Zacatecas pasó de 113 en 1998 a 25 en 2011; y Oaxaca que hasta 2011 contaba con 74 organizaciones (SRE, 2011). ${ }^{2}$

Dichas asociaciones se han concentrado, principalmente, en los estados de Arizona, Texas, Illinois y California; este último es el estado que más organizaciones de migrantes alberga: 783 entre clubes y federaciones, seguido de Texas con 483, Illinois con 135 y Arizona con 51 organizaciones. En lo que respecta a los migrantes michoacanos, han logrado consolidar fuertes lazos con sus comunidades de origen a través de estos clubes de oriundos. Por ejemplo, 76 de los 122 clubes de migrantes michoacanos están concentrados en California, en particular en el área metropolitana de Los Ángeles se encuentran 22 organizaciones. En muchos casos se trata de agrupaciones más bien informales, sólo conocidas por sus miembros y con escaso contacto con otros grupos de México y Estados Unidos, lo que dificulta saber con exactitud cuántos son y dónde están. Otro aspecto es que precisamente el surgimiento y consolidación de los clubes de migrantes permitió estructurar un siguiente nivel organizativo, las federaciones, que son organizaciones conformadas por clubes de migrantes provenientes de un mismo estado de origen en México, así como las confederaciones, que son metaorganizaciones ${ }^{3}$ conformadas por federaciones de migrantes provenientes de

${ }^{2}$ Es importante destacar que los datos antes mencionados deben tratarse con cautela ya que las fuentes a través de las cuales se elaboró esta información presentan ciertas limitaciones e inconsistencias. Por ejemplo, la información es exclusivamente de clubes de migrantes, sin embargo, se pudo corroborar que también incluyen a las federaciones de migrantes, por eso en ocasiones los datos están sobre-representados.

${ }^{3}$ En la teoría organizacional, una meta-organización es aquella organización cuyos miembros son organizaciones, no individuos, dichos modelos son cada vez más comunes en el ámbito internacional e incluso son herramientas importantes para establecer reglas transnacionales. Otro aspecto importante es que son relativamente fáciles de crear porque no requieren muchos recursos iniciales y son organizaciones que albergan más fuentes de conflicto y tensión que las organizaciones conformadas por individuos (Ahme y Brunsson, 2005). 
diferentes regiones en México. Dichas organizaciones tienen como propósito fortalecer los vínculos sociales entre los clubes y organizaciones en ambos lados de la frontera para promover proyectos filantrópicos en las comunidades de origen.

El envío de remesas por los migrantes es uno de los temas más trabajados en los últimos años. Diversos estudios coinciden en que estos recursos constituyen el impacto más notorio y tangible de la migración internacional en los países de origen (Papail y Arrollo, 2004). Sin embargo, estas organizaciones recaudan y envían otro tipo de remesas a las comunidades natales conocidas como remesas colectivas que, a diferencia de las remesas familiares o individuales, cobran un significado específico si se les distingue por su origen y destinatario, además de otros usos cualitativos de importancia, como la función y los montos enviados. ${ }^{4}$ En ese sentido, las remesas colectivas son un donativo sin fines de lucro exclusivo para financiar proyectos o bienes colectivos en la comunidad de origen (Duquette-Rury, 2011; Goldring, 2005; Orozco y Welle, 2005; Bada, 2004b).

Este tipo de remesas también es uno de los principales factores para el surgimiento de mecanismos de cooperación entre los migrantes organizados y el gobierno mexicano para fomentar el desarrollo y bienestar de sus pueblos de origen, además de reafirmar su identidad y conservar el sentimiento de pertenencia hacia las comunidades donde vivieron su proceso original de socialización (González Gutiérrez, 2006b).

Isomorfismo institucional: el arte de comprender a las organizaciones

Si bien existen diversos vocabularios para describir las características que poseen las organizaciones, el nuevo institucionalismo ha ganado terreno como perspectiva teórica para conocer más sobre los procesos de homogeneización y de cambio institucional en las organizaciones, particularmente

4 Para un estudio más amplio sobre el impacto económico de las remesas véanse, por ejemplo, los trabajos de Canales (2008), Papail y Arrollo (2004) y Goldring (2004, 2005).

$98 \frac{\text { PRIMER SEMESTRE } 2014}{\text { MIGRACIÓN Y DESARROLLO NÚM. } 22}$ 
a través del concepto de isomorfismo como tendencia reductora de la diversidad organizacional. Los precursores de esta teoría sostienen que los modelos organizativos, una vez que se institucionalizan, se hacen difusos, lo que permite que las estructuras organizativas sean más parecidas entre sí (Beckter, 2010; Scott, 2005; DiMaggio y Powell, 1999; Meyer y Rowan, 1999; Brunsson y Olsen, 1993).

En este sentido, los modelos organizativos son el conjunto de prácticas, rutinas, procedimientos o técnicas que una organización implementa para el logro de objetivos y metas relativamente estables, ya sea a través de una estrategia planeada o bien como consecuencia de su propia inercia en la búsqueda de soluciones a problemas concretos (Montaño Hirose, 2001; DiMaggio y Powell, 1999). De hecho, a través de los modelos organizativos se pueden apreciar los lenguajes, símbolos y significados que comparten los diferentes miembros y que le dan sentido e identidad a la organización, al tiempo que moldean significativamente la escala de acción. ${ }^{5}$

No obstante, los modelos organizativos prevalecientes en una organización pueden ser atribuidos no sólo a la complejidad de las relaciones a su interior o a los requerimientos estructurales, sino más bien a la existencia de ciertos factores organizacionales como los siguientes: a) los llamados «mitos racionales», que suelen constituir el meollo de los problemas en los sistemas de valores y creencias que comparten los miembros de la organización; $b$ ) el tipo de control, que explica la forma en que la organización coordina sus actividades; c) el desempeño de la organización, que generará legitimidad; y d) el estilo de liderazgo que predomina en la organización (Mintzberg, 1995; Hall, 1983; Meyer y Rowan, 1999; Scott, 2001; Brunsson y Olsen, 1993; Rombach, 1993).

De esta manera, en el nuevo institucionalismo se parte del supuesto de que las organizaciones, para sobrevivir, buscan legitimidad en contextos complejos en los que interactúan con otras organizaciones aparentemente

${ }^{5}$ La identidad organizacional es entendida como el conjunto de características simbólicas que los miembros de una organización consideran dominantes, distintivas y perdurables, a través de las cuales los procesos de reapropiación de modelos de organización guardan una estrecha relación de efecto mutuo (Ríos Ibarra, 2003). 
similares con las que intercambian información, recursos sociales y materiales, y que en algún momento determinarán el tipo de actividades a desempeñar, así como su comportamiento organizacional (Beckter, 2010; Zucker, 1999).

El isomorfismo institucional es, pues, un concepto que explica el proceso a través del cual las organizaciones se vuelven más homogéneas unas con respecto a otras al enfrentar condiciones contextuales aparentemente similares (DiMaggio y Powell, 1999). Este proceso parte de la premisa de que las organizaciones, al incorporar elementos organizativos supuestamente legitimados al exterior, que definen el valor de los elementos estructurales, promueven el éxito y la supervivencia de la organización, reducirán la incertidumbre y las posibilidades de fracaso (Montaño Hirose, 2010).

La homogeneización, a través de la cual es posible transferir modelos organizativos, puede darse por medio de tres formas de isomorfismo: mimético, coercitivo y normativo, las cuales operan detrás de los procesos de cambio institucional (DiMaggio y Powell, 1999). Se supone que los modelos organizativos, al ser efectivos en organizaciones que comparten el tiempo, espacio y otras características organizacionales aparentemente similares, funcionarán de la misma manera en aquellas organizaciones que los adoptan y adaptan a sus estructuras (DiMaggio, 1999; Scott y Meyer, 1999; Green y Li, 2011; Ríos Ibarra, 2003).

En este sentido, los sociólogos organizacionales con frecuencia encuentran que las conductas y estructuras que son institucionalizadas en la organización cambian más lentamente que las que no lo son, pues «las instituciones no sólo limitan las opciones: establecen los criterios por los que las personas descubren sus preferencias, en otras palabras, algunos de los costos hundidos más importantes son los cognoscitivos» (DiMaggio y Powell, 1999: 45).

Los clubes de migrantes mexicanos en Estados Unidos, que desde perspectivas tradicionales de estudio podrían ser considerados como instancias organizativas informales, que incluso no cumplen con los requisitos estructurales de una organización, desde la óptica del nuevo institucionalismo y, por supuesto, de la teoría organizacional, pueden ser definidas como estruc-

$100 \frac{\text { PRIMER SEMESTRE } 2014}{\text { MIGRACIÓN Y DESARROLLO NÚM. } 22}$ 
turas informales que, sin embargo, logran tener influencia en la conformación, persuasión y modelaje de estructuras formales al institucionalizar sus prácticas, rutinas y procedimientos organizacionales. En otras palabras, las organizaciones formales e informales transitan de lo normativo a lo cognoscitivo, de los compromisos a las rutinas, de los valores a las premisas, de la motivación a la lógica del seguimiento de las reglas como una evolución de sus procesos organizativos (Meyer y Rowan, 1999).

\section{Modelos organizativos DE los oriundos Michoacanos EN Estados Unidos}

Aun cuando la estructura en las organizaciones ha sido estudiada desde distintas perspectivas que se enfocan en sus componentes y las relaciones con el contexto, dicha estructura también puede ser estudiada a partir de la división de las actividades y la forma en que éstas se coordinan para alcanzar objetivos específicos. En las siguientes líneas explicaremos la diferencia en materia de modelos organizativos de los clubes michoacanos en Los Ángeles que conformaron nuestro estudio y que permiten identificar el conjunto de prácticas, rutinas y procedimientos organizacionales a través de las cuales se dividen las tareas y actividades al interior del club, obteniendo así la coordinación y control de las mismas.

Los clubes de migrantes, como organización, se establecen como una fuerza que influye en la vida y comportamiento de sus membresías. Es decir, pertenecer a este tipo de organizaciones es un indicador de la importancia que tienen los valores y el sentido de pertenencia al lugar de origen. En este sentido, uno de los primeros hallazgos entre los clubes michoacanos examinados es el hecho de que los comportamientos y las actitudes de los que participan en ellos se han modificado paulatinamente. Esto es el resultado de la gradual adopción y adaptación de una serie de valores, prácticas y rutinas que de otro modo probablemente no habrían podido conocer si no es a través de su participación en estas asociaciones. 
Otra característica importante es el sentido de colectividad que generan al involucrar a todos los miembros del club en las distintas actividades, sin importar el estatus social o el puesto que desempeñen en la organización. Ciertamente, el argumento anterior ilustra uno de los fines de toda organización, sea social, política o de otra índole: buscar convencer o inducir a los miembros que la conforman para alcanzar objetivos y metas comunes. En otras palabras, la mejor forma de organización será aquella que asegure la cooperación de los miembros mediante un trabajo eficiente con beneficios recíprocos (Scott, 2001; Montaño Hirose, 2001).

Si bien es cierto que los clubes de migrantes son sistemas colectivos donde las actividades que se realizan son controladas y coordinadas, también desarrollan elementos culturales para definir características y establecer diferencias con respecto a otras organizaciones de migrantes. Esta forma organizativa ha motivado el surgimiento de otras nuevas organizaciones que incorporan prácticas y procedimientos organizacionales aparentemente efectivos y exitosos en las ya existentes, que les permiten conseguir legitimidad, prestigio y supervivencia organizativa (Brunsson y Olsen, 1993; Zucker, 1999).

Analizar la estructura organizativa de los clubes de migrantes a partir del caso de los clubes michoacanos en Los Ángeles resulta particularmente útil, ya que dicha estructura influye, a través de una serie de factores, en el comportamiento y en la capacidad de acción de sus miembros. La evidencia empírica que algunos estudios previos han presentado se limita a explicar únicamente aspectos limitados al respecto, como por ejemplo el número de integrantes que las conforman o el tipo de liderazgo que predomina. Si bien estas dimensiones son parte importante de la estructura, no son suficientes para hacer afirmaciones sobre su desempeño organizativo, mucho menos para explicar las dinámicas internas por medio de las cuales se producen resultados y se alcanzan objetivos en las organizaciones.

La estructura organizativa en los clubes examinados se puede entender como la distribución de los miembros de la organización en los diferentes puestos que la conforman, donde cada uno lleva a cabo distintas tareas que están especificadas en reglas y normas de acuerdo con el nivel jerárquico. En 
otras palabras, una implicación de esta definición será la división del trabajo, que en el caso de los clubes de migrantes michoacanos consiste en la conformación de la «mesa directiva». El siguiente testimonio del presidente del Club Jiquilpan USA nos sirve de ejemplo:

Legalmente estamos formados como cualquier agrupación, con presidente, vicepresidente, tesorero, subtesorero, secretario, sub-secretario, vocales, entre otros. Pero prácticamente todos desempeñamos un puesto en el club. Incluso, hace poco formamos la nueva mesa directiva, todos de común acuerdo [...] Yo como presidente, tengo que estar al tanto de que se lleven a cabo todas las actividades, tal como las planeamos (Presidente, Club Jiquilpan USA, 2012).

La forma en la que están conformadas las mesas directivas del resto de los clubes analizados es similar a la que presenta el Club Jiquilpan, solamente varían en el número de puestos. Una característica adicional respecto a la estructura organizativa es que ésta no es fija a lo largo del tiempo, por el contrario, es modelada en cuanto a los puestos que se tienen y el tiempo por el que se designan.

No obstante, la estructura organizativa está determinada por una serie de dimensiones o factores que la teoría organizacional considera importantes para identificar las dinámicas internas en la organización: 1) el tamaño; 2) la formalización; 3) la centralización de la toma de decisiones; y 4) la complejidad organizativa (Hall, 1983; Mintzberg, 1995). Dichas dimensiones, además de condicionar el comportamiento de los individuos en la organización, también son un medio para lograr objetivos y metas colectivas. ${ }^{6}$

De la estructura a los modelos organizativos

En nuestro estudio se identificaron tres tipos de estructuras organizativas en los clubes migrantes michoacanos: horizontales, verticales y diversifica-

6 Para una explicación más detallada sobre cada uno de estos factores estructurales entre los clubes michoacanos en Estados Unidos, véase González Zepeda (2012). 
das o profesionales. Esta tipología (véase el cuadro 1) parte de la premisa de que los clubes michoacanos compiten entre sí por la búsqueda de recursos debido, entre otras cosas, a las propiedades de la estructura organizativa de cada uno. Es decir, gran parte de la conformación de los modelos organizativos deriva de reemplazar una forma organizacional por otra, configurando con ello la identidad, legitimidad, prestigio y, por supuesto, sobrevivencia organizacional.

Dentro de esta tipología también fue posible identificar tres estilos de liderazgo. En la teoría organizacional el liderazgo es una forma especial de poder que se basa en las capacidades personales de un individuo para inducir la aceptación voluntaria de los miembros de la organización (Hall, 1983; Mintzberg, 1995; Drucker, 2006; Handy, 2006). El liderazgo se distingue del concepto de poder; el primero supone el cambio de preferencias a partir de la persuasión, mientras que el segundo sólo implica que las preferencias de los sujetos se mantienen en incertidumbre. Entonces, el líder es una influencia sobre lo que los miembros de la organización hacen y piensan.

No obstante, en los clubes michoacanos las funciones del liderazgo suponen: 1) la definición de la misión y el papel que juega el club en ambos lados de la frontera; 2) la incorporación del propósito de la organización y la selección de los medios para alcanzar los fines deseados de manera colectiva; y 3) la integridad de la organización, es decir, aquí se mezclan los valores y las relaciones públicas que logren establecer en el contexto transnacional para que se acepten y legitimen las decisiones. En este sentido: «La persuasión de los individuos y la innovatividad en ideas y toma de decisiones es lo que hace la diferencia entre liderazgo y la simple concentración de poder» (Hall, 1983: 158 [cursivas del original]).

$104 \frac{\text { PRIMER SEMESTRE } 2014}{\text { MIGRACIÓN Y DESARROLLO NÚM. } 22}$ 


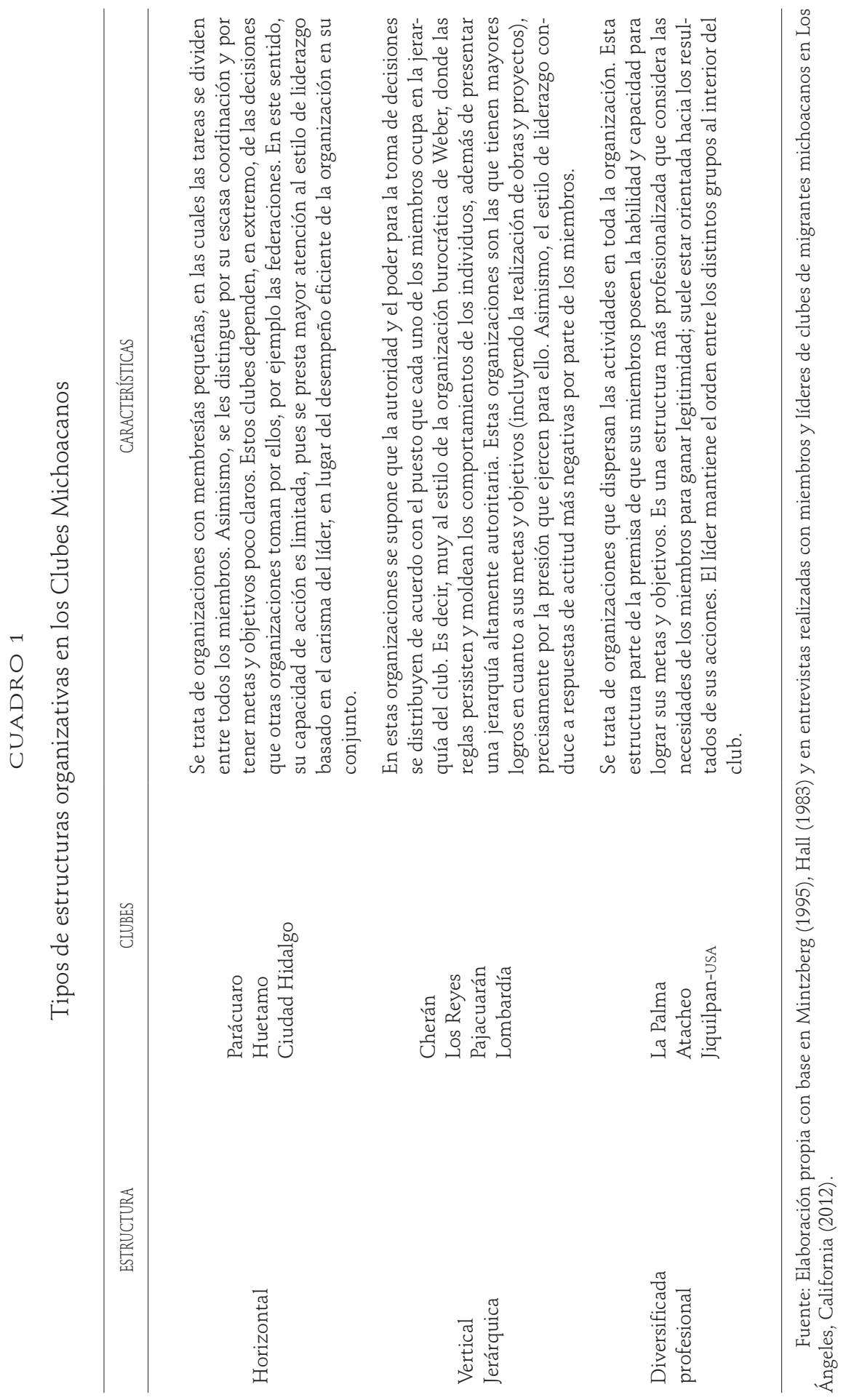


Como se puede apreciar en el cuadro anterior, las estructuras horizontales se caracterizan por la falta de elaboración y coordinación en los puestos de la mesa directiva, los cuales dependen de la ayuda y decisiones que la federación a la que pertenecen pueda tomar por ellos. Por otro lado, las estructuras verticales se caracterizan porque todas las reglas a las que se apegan los miembros para llevar a cabo sus actividades penetran en toda la organización, donde se fomenta la comunicación formal en todos sus niveles. La toma de decisiones se concentra, exclusivamente, en la cadena de autoridad formal que se encuentra en la cúspide de la mesa directiva.

Finalmente, en las estructuras profesionalizadas la normalización de las habilidades de los miembros es fundamental para lograr la coordinación de actividades, pues parten de que sus membresías son creativas, innovadoras y que aprenden conforme se les motiva para participar en las decisiones colectivas. Estos clubes cuentan con el apoyo de otras organizaciones (ya sean de migrantes o no) que les asesoran para mejorar su rendimiento y el desempeño de la organización; pero para adoptar las recomendaciones, primero evalúan su capacidad y la de sus miembros antes de modificar cualquier comportamiento o procedimiento organizacional.

Apropiación de modelos organizativos: buscando un ideal

La transferencia de modelos organizativos se ha convertido en un fenómeno muy frecuente en el universo organizacional. Esta situación se puede observar en los clubes de migrantes michoacanos examinados, que constantemente están buscando la forma de ser más eficientes, generar más recursos, llevar a cabo más obras y proyectos en la comunidad de origen y aumentar su membresía. Esta necesidad, por ser organizaciones legítimas y exitosas en el campo organizacional en el que se desarrollan, los lleva a generar una serie de vínculos con otras organizaciones migrantes como las federaciones, confederaciones e incluso con clubes de otras latitudes con los que establecen alianzas de cooperación.

$106 \frac{\text { PRIMER SEMESTRE } 2014}{\text { MIGRACIÓN Y DESARROLLO NÚM. } 22}$ 
De esta forma, la transferencia de modelos organizativos puede ser una opción viable para los clubes michoacanos que apenas inician su trayectoria organizativa y buscan solucionar la problemática de eficiencia y efectividad. Pero también es una oportunidad para organizaciones con trayectoria más larga para refrendar su prestigio, legitimidad y éxito, un aspecto que ciertamente les preocupa. En ese sentido, podemos decir que el proceso de reapropiación de modelos organizativos en los clubes de migrantes michoacanos es aquella serie de factores, interacciones, negociaciones y reinvención de significados que intervienen en la adopción, consolidación o rechazo de un modelo organizativo por los miembros de la organización receptora.

\section{DesarRollo local E ISOMORFISMO INSTITUCIONAL}

Así como las organizaciones están institucionalizadas, en la medida en que su comportamiento está determinado por reglas culturalmente condicionadas, también reflejan valores, intereses, opiniones y expectativas relativamente estables de cada miembro. En este apartado, partimos de la premisa de que cada club de migrantes tiene una historia y que con el paso del tiempo desarrolla sus propias ideas acerca de qué tareas son más importantes, qué resultados son mejores y cómo pueden lograrse dichos resultados.

En su vida organizativa, es muy común la incorporación de elementos institucionalizados y racionalizados, principalmente modelos y estructuras organizativas legitimadas y efectivas que son parte del mismo campo organizacional y que también dan pie al surgimiento de nuevas organizaciones que tienen necesidad de eficiencia en las acciones que emprenden. Asimismo, buscan mecanismos que garanticen la legitimidad de la organización, por ejemplo, a través de la interacción con otros clubes en las federaciones, o bien en los distintos eventos a los que acuden y donde les es posible observar «la mejor manera de hacer las cosas». El siguiente testimonio ejemplifica el argumento anterior:

La ventaja que tenemos al estar en la Federación [Michoacana] es que ahí podemos obtener mucha información que después utilizamos para despertar más 
conocimientos. Pero más que nada nos permite conocer a otras mesas directivas con las que tenemos la oportunidad de intercambiar ideas que nos abren los ojos, porque tenemos mucha ignorancia sobre varias cosas. Hoy ya estamos más informados y nos salen mejor las cosas porque vemos y hacemos lo que hacen otros clubes, pues siempre puedes aprender (Presidente, Club Los Reyes en Los Ángeles, California, 2012).

Por lo tanto, los elementos que llegan a incorporar los miembros de los clubes a sus estructuras organizativas son racionales y efectivos, pues reducen la incertidumbre y mantienen la confianza que la organización requiere. Sin embargo, el club que incorpora dichos elementos debe tomar en consideración que su actividad práctica debe mantener a la organización en un estado de integración flexible al interior, es decir, que los miembros que incorporan los elementos de otras organizaciones los adopten y adapten a los requerimientos de sus actividades organizativas $y$, por supuesto, de sus habilidades personales.

Cambio institucional: la metamorfosis de los clubes de migrantes

Desde el enfoque del nuevo institucionalismo, es posible comprender y explicar el cambio organizacional en los clubes michoacanos vinculado a alguno de los siguientes factores: los intereses de la organización, la reintroducción de poder en la toma de decisiones, el riesgo y la incertidumbre organizacional y la legitimidad, éxito e isomorfismo institucional (Meyery Rowan, 1999; DiMaggio, 1999; Scott, 1994). Para los propósitos de este trabajo, nos enfocamos en el cambio organizacional producto de la legitimidad, éxito e isomorfismo institucional, ya que éste se acopla mejor al análisis del funcionamiento de los modelos organizativos observado en los clubes de migrantes examinados.

Una de las principales características del cambio organizacional es que permite asignar un peso específico a la capacidad de las instituciones para determinar el pensamiento y la acción de los miembros de una organización.

$108 \frac{\text { PRIMER SEMESTRE } 2014}{\text { MIGRACIÓN Y DESARROLLO NÚM. } 22}$ 
En otras palabras, aquellas prácticas, rutinas y demás procedimientos organizacionales que llevan a cabo los clubes michoacanos como kermeses, rifas, bailes, colectas, incluso el tipo de obras y proyectos que apoyan en la comunidad de origen, al estar tan enraizados en las acciones de la organización, llegan a institucionalizarse, es decir, se dan por hecho y definen lo que tiene significado para la organización y las acciones que son posibles llevar a cabo. Así, el cambio organizacional ocurre como resultado de los procesos que hacen a los clubes michoacanos más similares, aunque no necesariamente más eficientes (González Zepeda, 2012).

El concepto de isomorfismo institucional nos permite explicar el proceso de homogeneización en los clubes michoacanos y la estabilidad y persistencia de los componentes institucionalizados (prácticas, rutinas y estructuras). Recordemos que este proceso ocurre a través de los siguientes tres mecanismos por medio de los cuales se disminuye la diversidad organizacional, es decir, se explicará la homogeneidad y no la variación organizativa.

Isomorfismo mimético: Se encontró un comportamiento de este tipo en tres de los clubes michoacanos analizados, que se caracterizan por ser organizaciones que no suelen estar activas constantemente y donde uno de sus mayores problemas es la falta de nuevos miembros que trabajen para llevar a cabo la misión de la organización. En las entrevistas realizadas con los representantes de estas organizaciones (los clubes Parácuaro, Huetamo y Ciudad Hidalgo), se corroboró que varias de las actividades que realizaban como club fueron producto de imitar lo que los paisanos de otras latitudes hacían. Por ejemplo, estos clubes reconocían el éxito que tenían las asociaciones de inmigrantes zacatecanos y jaliscienses en California, de quienes retomaron varias ideas, formas y modelos de cómo hacer las cosas. El siguiente segmento ilustra este punto:

Prácticamente nuestra organización es la misma que las de otros estados, lo único que las hace diferentes es la gente y la responsabilidad de la gente, porque tal vez no supimos escoger de todo lo que se observa en otros clubes, pensando en que la gente lo puede hacer, y aunque digan que sí, lo cierto es que a la hora del trabajo ya no es lo mismo porque no es lo mismo hablar que ejercitar lo que 
se habla, pero el caso es que somos igual a otros clubes (Presidente, Club Ciudad Hidalgo en Los Ángeles, 2012).

En este sentido, el isomorfismo mimético es la forma de imitación más simple que tienen los clubes examinados para reaccionar a la incertidumbre con respecto a los efectos de las reglas institucionales que imperan en el campo organizacional en el que interactúan. Estos efectos pueden ser las exigencias de la Federación Michoacana a la que pertenecen o incluso las presiones que puedan surgir de las oficinas consulares a donde los clubes acuden si desean participar en alguno de los programas gubernamentales. La mayoría de las veces la imitación tiene que ver con el deseo de participar en programas como el $3 \times 1$, donde otros clubes han legitimado sus estructuras y han podido realizar organizaciones exitosas.

Aquellos clubes de los cuales se toma el modelo organizativo «legitimado y exitoso» pueden no estar conscientes de ello o pueden desear no ser imitados, pero para el caso esto sirve como una fuente conveniente de prácticas que la organización que las retoma puede usar. Un ejemplo común entre los clubes de migrantes mexicanos en Estados Unidos es la organización de kermeses para recaudar fondos: a pesar de que nadie a ciencia cierta sabe a quién o a qué clubes se le ocurrió la idea de implementar esta actividad, dichas asociaciones durante años han imitado a otras en la realización de éstas y otras actividades para recaudar recursos.

Si los efectos de las actividades del club no son racionalmente los esperados - y aquí entra la cuestión del desempeño organizativo-, entonces la imitación de modelos institucionalizados y legitimados en el ámbito organizacional pueden compensar esta situación. Por un lado, la imitación proviene de la iniciativa que tiene el club por emprender y crecer, pero en estos casos se necesita más que eso, y dependerá entonces de la capacidad de decisión y acción de quien adopta y trata de adaptar el modelo organizativo. Por otro lado, la imitación puede convertirse en un escudo protector para la organización en caso de que fallen las decisiones (Beckter, 2010), pues se parte de la premisa de que el club hace lo que otros han hecho en su posición y han logrado el éxito.

$110 \frac{\text { PRIMER SEMESTRE } 2014}{\text { MIGRACIÓN Y DESARROLLO NÚM. } 22}$ 
Isomorfismo coercitivo: La homogeneización entre los clubes michoacanos es también el resultado de las presiones formales e informales que ejercen otras organizaciones que se encuentran en el mismo campo organizacional, como las federaciones y confederaciones, para que adapten directa o indirectamente estructuras y modelos. Esto con el fin de acceder a ciertos recursos deseados por la organización, pero que dependen del cumplimiento de las reglas impuestas por aquella organización superior. Un ejemplo que ilustra el argumento anterior es el siguiente testimonio:

Como a los clubes todavía les falta tener más visión y decisión, la Cofem (Consejo de Federaciones Mexicanas en Norteamérica) tiene las herramientas para entrenarlos como organizaciones, pues muchas veces no saben lo que quieren, entonces esa es una debilidad en la que tenemos que trabajar. Se trata de hacer clubes más capaces, porque somos una organización muy reconocida a nivel nacional en Estados Unidos que bajamos fondos para los clubes, pero también tenemos una mesa directiva que trabaja y entiende muy bien su labor como organización, algo que los clubes aún no entienden. Entonces el reto es que nuestras mesas directivas, que nuestros presidentes y nuestros clubes realmente se preparen y sigan nuestro ejemplo como organización (Vicepresidente COFEMClub Los Reyes, Los Ángeles, 2012).

Sin embargo, el ejercicio formal e informal de presión que ejercen otras organizaciones es más bien un mecanismo que conduce a la homogeneización de los clubes michoacanos con respecto a la dirección en la que el poder influye en el desarrollo organizacional, al menos eso es lo que se pudo corroborar en el estudio. En ese sentido, se identificaron aquellas situaciones que indicaban que las funciones de los clubes se debían a las presiones provenientes principalmente de las federaciones donde participaban, y en algunos casos también de las presiones ejercidas por instancias gubernamentales como el Consulado mexicano en Los Ángeles y la oficina de la Secretaría de Desarrollo Social (SEDESOL), con el fin de que los clubes adoptaran un modelo organizativo que les permitiera beneficiarse de los programas y recursos disponibles.

En este tipo de isomorfismo, el cambio se produce cuando los clubes han perdido credibilidad moral o funcionalmente, y al mismo tiempo cuando 
hay algún actor poderoso que es capaz de hacer cumplir un nuevo diseño organizacional. Esta situación se puede apreciar en el caso de los clubes Los Reyes, Cherán y Lombardía, que se caracterizan por ser estructuras rígidas y centralizadas, y donde solamente la voz de unos cuantos es la que se toma en cuenta. Asimismo, estos clubes se caracterizan por tener al frente a un líder con un estilo autoritario que basa la legitimidad de la organización en el control y la sanción.

Isomorfismo normativo: En este caso, el cambio es producto de la profesionalización de la organización, es decir, está motivado por la expectativa de lograr mejores resultados después de la adopción del modelo organizativo. Por lo tanto, las membresías de estos clubes no están sujetas a la presión para adoptar un modelo, pero sí son motivados desde el interior de la organización para buscar una solución organizacional específica a los problemas de este tipo. El siguiente segmento ilustra este punto:

Nosotros aprendemos de estar viendo lo que hacen los demás clubes y luego, pues uno le echa de su propia cosecha, y los resultados no los dejas esperar, los adaptas a las necesidades del club, porque lo que veas en un grupo zacatecano o jalisciense no te va a servir del todo en un grupo de Michoacán, y viceversa. Entonces tienes que hacer ajustes de acuerdo a la capacidad del grupo y el tipo de gente que participa en el club [...] (Vicepresidente, Club Jiquilpan USA en Los Ángeles, 2012).

Una vez que los modelos organizativos se han institucionalizado — como ocurre, por ejemplo, con los clubes zacatecanos en Estados Unidos, por su volumen y vasta trayectoria-, los clubes michoacanos estudiados pueden adoptar y utilizar dichos modelos de otros grupos como plantillas para el diseño de sus estructuras organizativas. Esto requiere estar al tanto de que su capacidad organizativa y las habilidades de los miembros de la organización son las más adecuadas para que el modelo pueda dar solución a sus problemas o demandas organizativas. Por lo tanto, un factor que contribuye a la transformación normativa es que los clubes de migrantes, al adoptar modelos y prácticas organizacionales que ya han sido probados en otros

$112 \frac{\text { PRIMER SEMESTRE } 2014}{\text { MIGRACIÓN Y DESARROLLO NÚM. } 22}$ 
clubes, a partir de los resultados, pueden decidir si los incorporan a sus estructuras o no. Este es un incentivo para que los miembros de la organización busquen mejores prácticas organizacionales.

Esta situación se pudo observar en el Club Jiquilpan USA, que al ser una organización que ha pasado por diferentes etapas, se ha mantenido como uno de los clubes con mayor estabilidad organizacional en comparación con el resto de los que conforman el estudio. Y esto se debe a que desde su formación ha experimentado diferentes maneras de hacer las cosas, ya sea a través de las experiencias que los miembros de la mesa directiva traen a la organización, o bien a través de lo que observan en clubes de otros orígenes. Incluso sus miembros consideran que fue muy difícil conformar esta organización, ya que mucha de la gente que empezaba a trabajar con ellos no confiaba en las acciones que el club podría emprender.

Otros clubes michoacanos que se pueden tipificar como organizaciones que transitan por el proceso de isomorfismo normativo son los clubes Atacheo y La Palma. En estas asociaciones, las redes vinculadas a la organización, por ejemplo la representación del club en otras ciudades de los Estados Unidos, son una herramienta clave para difundir la serie de normas, prácticas y rutinas a través del país de destino de los migrantes y hasta en las localidades de origen. Estas comunidades, con quienes frecuentemente mantienen el contacto y son parte de la estructura organizativa del club, contribuyen a la obtención de perspectivas más homogéneas en todos los niveles organizativos, a través de los cuales es posible resolver problemas con soluciones más adecuadas a su capacidad organizativa.

Por lo tanto, el isomorfismo normativo en estos clubes michoacanos se puede apreciar como un tipo de presión que aparece principalmente en aquellas organizaciones que son más profesionales, y que tienen una estructura organizativa más elaborada y flexible. Es decir, se trata de una estructura descentralizada, donde la autoridad en la toma de decisiones aparece en varios puntos de la organización. Esto hace más fácil la incorporación de las opiniones de los miembros del club, quienes al tener cierto grado de autonomía en las actividades que realizan pueden innovar la forma en que llevan a cabo sus labores y beneficiar a la organización en su conjunto. 
En síntesis, los mecanismos que se han explicado en esta sección se expresan como estilos de acción y cambio en organizaciones con antecedentes diferentes que se sienten atraídas por los distintos modelos organizativos que están presentes en su campo organizacional. Es decir, la atracción hacia los modelos organizativos difiere a pesar de las similitudes generales que puedan presentar en las situaciones a las que se enfrentan. Por lo tanto, se considera que estos procesos de isomorfismo no son suficientes para explicar el grado en que las organizaciones se han hecho más similares estructuralmente.

Los nexos entre modelos organizativos y desarrollo local

Actualmente es difícil saber con exactitud cuántos clubes michoacanos en la región de Los Ángeles participan en el Programa 3×1, ya que en la mayoría de los casos se trata de clubes que se conforman para llevar a cabo una obra específica en el pueblo de origen, y al concluirla dejan de trabajar en el programa sin perder su registro en el padrón. Los clubes michoacanos que participan constantemente en este Programa, según la información proporcionada por la representación de SEDESOL en Los Ángeles, son los que tienen mayor antigüedad, como en el caso de Los Reyes, Lombardía, Jiquilpan, Pajácuarán e incluso Cherán.

Sin embargo, también participan clubes que han ido apareciendo en los últimos años y que son motivados por las autoridades en los municipios de origen que, al saber de la existencia del Programa y de los beneficios que pueden adquirir, incentivan a los paisanos a conformar estas organizaciones, con el fin de realizar alguna obra que beneficie a la comunidad, sobre todo en materia de infraestructura social básica.

Estas decisiones que se toman para realizar algún proyecto u obra surgen de las presiones a las que es sometido el modelo organizativo del club (miméticas, coercitivas y normativas), y que llevan a los clubes a cambiar sus metas y desarrollar nuevas prácticas que pueden adquirir un valor más allá de los requisitos técnicos de la obra o proyecto del que se trate. Un ejemplo que nos permite entender las repercusiones en la estructura de los clubes

$114 \frac{\text { PRIMER SEMESTRE } 2014}{\text { MIGRACIÓN Y DESARROLLO NÚM. } 22}$ 
michoacanos al adoptar y adaptar un modelo organizativo que no se adecúa a los requerimientos reales de la organización ni a las capacidades y habilidades de la membresía se puede apreciar en el siguiente testimonio:

Cuando iniciamos con el club Huetamo nos reuníamos mucho con la gente de Lombardía que ya tenía su club funcionando, ellos ya estaban bien organizados. Nosotros veíamos cómo hacían muchos proyectos en Michoacán, fue cuando nos invitaron a unirnos en la Federación Michoacana Lázaro Cárdenas, el presidente fue el que nos dijo cómo hacer las cosas, él era también presidente del Club Lombardía. Entonces empezamos a hacer todo lo que ellos nos decían: organizar a la gente, hacer eventos, reunirnos y un montón de cosas que si no se hacían, pues no nos incluían para los proyectos. Al final hicimos todo lo que nos dijeron las gentes de Lombardía, le metimos al $3 \times 1$ y los proyectos productivos y nomás fue pura pérdida de dinero, ni negocio, ni ganancias, ni nada, al final ya no siguió el club, la gente se desanimó (Presidente, Club Huetamo en Los Ángeles, 2012).

Existe una gran diversidad en el tipo de proyectos que los clubes michoacanos pueden llevar a cabo en las comunidades de origen a partir del Programa $3 \times 1$. Se identificaron casos de clubes que están enfocados exclusivamente en realizar obras y proyectos sociales entre los sectores más vulnerables en la comunidad de origen, como son los clubes de Cherán, Jiquilpan, La Palma e incluso Atacheo. Lo que llama la atención es que se trata de clubes que se habían clasificado como diferentes en el tipo de estructura y modelo organizativo. Por ejemplo, el Club Cherán, que figuraba como una organización centralizada y rígida, con un modelo organizativo adoptado de manera coercitiva, es uno de los clubes que más proyectos de infraestructura ha financiado por medio del $3 \times 1$.

En cambio, los clubes de Jiquilpan, Atacheo y La Palma, con estructuras más flexibles y profesionalizadas, que podrían llevar a cabo más y mejores proyectos sociales vía el Programa $3 \times 1$, son los que menos tienen y han decidido trabajar por su cuenta hasta lograr mayor experiencia. Incluso al tener una capacidad organizativa relativamente estable, podrían estar inmersos en proyectos de mayor responsabilidad, como los productivos. Sin 
embargo, lo que se pudo corroborar es que precisamente al ser clubes más normativos, prefieren informarse al respecto, ver lo que ocurre con otros clubes y así decidir por qué ruta transitar. Esto no significa que el resto de los clubes michoacanos no hayan participado en algún momento en obras sociales, al contrario, prácticamente todos los clubes tienen la experiencia de haber realizado algún tipo de obra o proyecto social comunitario vía el Programa $3 \times 1$.

Por el contrario, están aquellos clubes que se enfocan en los llamados proyectos productivos, después de que por varios años estuvieron financiando obras y proyectos sociales en las comunidades de origen, aunque sus resultados son aún inciertos en esta nueva etapa. Los clubes que actualmente ponen toda su capacidad organizativa para llevar a cabo este tipo de proyectos son Los Reyes y Lombardía, caracterizados por ser organizaciones rígidas, centralizadas, y que buscan a toda costa imponer sus modelos en otras organizaciones; y Ciudad Hidalgo, que se categorizó como un club con una estructura simple y que proviene de un proceso de isomorfismo mimético.

En este sentido, la experiencia participativa de los migrantes michoacanos en el Programa $3 \times 1$ les ha permitido obtener cierto grado de madurez organizativa respecto al tipo de obras que realizan. Por lo tanto, el programa también ha influido en la personalidad y formación de líderes que intentan, junto con la organización, salir adelante en una sociedad que a veces puede ser hostil y crítica de sus actividades.

\section{Conclusiones}

El interés por examinar desde esta perspectiva a los clubes de migrantes michoacanos en los Ángeles, California, se basa en traer a colación el andamiaje de la teoría organizacional a los estudios sobre migración y formas asociativas migrantes, temas que durante años han sido estudiados desde otras perspectivas, con el fin de comprender de manera más precisa el funcionamiento y dinámica organizativa que subyace al interior de estas organizaciones. Nuestra atención se dirigió no sólo a los vínculos transnaciona-

$116 \frac{\text { PRIMER SEMESTRE } 2014}{\text { MIGRACIÓN Y DESARROLLO NÚM. } 22}$ 
les que estas asociaciones logran construir, o a señalar aspectos como el número de integrantes que conforman estas organizaciones, o bien el tipo de liderazgo que predomina, sino que también se centró en estudiar y comprender, desde la óptica de la teoría organizacional, la estructura y componentes fundamentales al interior de los clubes michoacanos y que les permiten funcionar como tales. Para ello, se examinaron los vínculos entre las organizaciones similares que interactúan en un mismo campo organizacional, así como entre aquellas que presentan diferencias estructurales o de modelos organizativos. De manera particular, se analizaron los vínculos horizontales y verticales que llevan a estas formas organizativas a volverse cada vez más homogéneas (en particular se examinó el proceso de isomorfismo institucional) y a la vez más complejas. También se analizaron las relaciones locales y no locales que dan origen a las acciones que emprenden estos clubes michoacanos, principalmente aquellas que se enfocan en promocionar el desarrollo de las comunidades de origen; y se consideraron los aspectos institucionales y organizacionales que dan origen a las prácticas y rutinas que durante décadas han caracterizado a estas formas organizativas.

En ese sentido, a lo largo de este trabajo se mostraron algunos aspectos relacionados a los modelos organizativos adoptados por dichos clubes de migrantes y la realización de obras y proyectos eficientes financiados con remesas colectivas en las comunidades de origen, mediante la apropiación de prácticas y rutinas organizativas. Para ello, se analizó la conformación de su estructura organizativa, lo cual resultó particularmente útil para identificar los factores que influyen en el comportamiento y en la capacidad de acción de sus membresías.

De esta manera fue posible identificar tres tipos de estructuras organizativas imperantes en estos clubes (horizontales, verticales y profesionales) que caracterizan su capacidad de acción y los distintos estilos de liderazgo que imperan en estas asociaciones migrantes mexicanas. Y si bien nuestro estudio ilustró que las organizaciones con estructuras profesionales presentan una más clara definición de su misión como tales, la cual encarna un propósito claro y realizable mediante normas y valores compartidos, así como la preservación de la integridad de la organización, también permitió advertir 
que esto no necesariamente conduce a la mayor promoción de formas de desarrollo local. De hecho, nuestros hallazgos mostraron que las estructuras verticales son las que tienen mayores logros en cuanto a sus metas y objetivos, incluyendo la realización de obras y proyectos en sus comunidades de origen.

Finalmente, la perspectiva analítica adoptada en este trabajo ilustra que los clubes de migrantes michoacanos (y de otras latitudes) no sólo son capaces de generar recursos y participar en la política social en las comunidades de origen en México, sino que también a través de sus formas organizativas se puede explicar y entender por qué algunas organizaciones se desempeñan mejor que otras, de ahí la importancia de examinar con detenimiento los procesos de reapropiación de modelos organizativos entre las organizaciones de migrantes. La adopción de dicha perspectiva permitirá apreciar de mejor manera la configuración de la identidad de cada organización, la legitimidad, el prestigio y, por supuesto, la sobrevivencia organizacional.

\section{Bibliografía}

Ahme, Góran y Niels Brunsson (2005), «La regulación suave desde una perspectiva organizacional», Gestión y Política Pública, vol. 14, núm. 3.

BADA, Xóchitl (2004b), "Clubes de michoacanos oriundos: desarrollo y membresía», Migración y Desarrollo, vol. 2, núm. 2.

(2003), «La participación cívica comunitaria transnacional de los clubes de michoacanos», en Gustavo López Castro (coord.), Diáspora michoacana, Zamora, Michoacán, El Colegio de Michoacán, Gobierno del Estado de Michoacán. BASHI, Vilna Francine (2007), Survival of the Knitted. Immigration Social Networks in a Stratified Worrld, Stanford, Stanford University Press.

BeCKTER, Jens (2010), "Institutional Isomorphism Revisited: Convergence and Divergence in Institutional Change», Sociological Theory, vol. 28, núm. 2.

Brunsson, Nils y Johan P. Olsen (1993), "Organizationals forms: Can we choose them?», en Nils Brunsson y Johan P. Olsen (eds.), The Reforming Organization, New York, Routledge.

$118 \frac{\text { PRIMER SEMESTRE } 2014}{\text { MIGRACIÓN Y DESARROLLO NÚM. } 22}$ 
Canales Cerón, Alejandro (2008), «Remesas y desarrollo en América Latina. Una relación en busca de teoría», Migración y Desarrollo, núm.11.

DiMaggio, Paul y Walter W. Powell (1999), «Introducción», en Paul DiMaggio y Walter W. Powell (comps.), El nuevo institucionalismo en el análisis organizacional, México, Fondo de Cultura Económica.

(1999), «Retorno a la Jaula de Hierro: El isomorfismo institucional y la racionalidad colectiva en los campos organizacionales», en Paul DiMaggio y Walter W. Powell (comps.), El nuevo institucionalismo en el análisis organizacional, México, Fondo de Cultura Económica.

Drucker, Peter (2006), El lider del futuro, Barcelona, Peter Drucker Fundation, Deusto.

DuguetTe-Rury, Lauren (2011), "The Welfare Effects of Migrant-State Coproduction. The $3 \times 1$ Program, Remittances and Public Goods Provision in Mexico", Prepared for presentation at Politics and Migration in Out-Migration Countries, Workshop, CIDE, México.

Escala RabadÁn, Luis, Gaspar Rivera-Salgado y Rigoberto Rodríguez (2011), «Is More Necessarily Better? Leandership and Organizational Deveplopment of Migrant Hometown Associations in Los Angeles, California», Migraciones Internacionales, vol. 6 , núm. 2 .

(2005), «Derechos Humanos y asociaciones de migrantes mexicanos en California», Migraciones Internacionales, vol. 3, núm. 2.

García Zamora, Rodolfo (2012), Crisis, migración y desarrollo. Los actores sociales y el reto de las nuevas políticas públicas en México, Zacatecas, Doctorado en Estudios del Desarrollo, Universidad Autónoma de Zacatecas.

(2005), «Las remesas colectivas y el Programa $3 \times 1$ como proceso de aprendizaje social transnacional», documento de contexto para presentarse en el seminario, La participación cívica y social de los migrantes mexicanos en Estados Unidos, Washington DC, Centro Internacional Woodrow Wilson.

Goldring, Luin (2005), "Implicaciones sociales y políticas de las remesas familiares y colectivas», en Raúl Delgado Wise y Beatrice Knerr (coords.), Contribuciones al análisis de la migración internacional y el desarrollo regional en México, México, UAZ, Miguel Ángel Porrúa.

(2004), «Family and Collective Remittances to Mexico: A Multi-dimensional Typology», Development and Change, vol. 35, núm. 4.

(2002), «The Mexican State and Transmigrant Organizations: Negotiating the Boundaries of Membership and Participation", Latin American Research Review, vol. 37, núm. 3. 
GonzÁlez Gutiérrez, Carlos (2006b), «Del acercamiento a la inclusión institucional: la experiencia del Instituto de los Mexicanos en el Exterior», en Carlos González Gutiérrez (coord.), Relaciones Estado-diáspora: aproximaciones desde cuatro continentes, Tomo I, México, SRE, IME, UAZ, ANuies, Miguel Ángel Porrúa.

(1995), «La organización de los inmigrantes mexicanos en Los Ángeles», Revista Mexicana de Política Exterior, núm. 46.

GonZÁlez ZepeDa, C. Alberto (2012), Isomorfismo institucional y promoción del desarrollo local en asociaciones de migrantes michoacanos en Los Ángeles, California, Tesis de Maestro en Desarrollo Regional, El Colegio de la Frontera Norte, México.

Green JR., Sandy Edward y Yuan Li (2011), «Rhetorical Institutionalims: Languague, Agency, and Structure in Institutional Theory since Alvesson 1993", Journal of Management Studies, vol. 48, núm. 7.

Hall, Robert (1983), Organizaciones: estructura y proceso, Madrid, Prentice Hall.

HANDY, Charles (2006), «El nuevo lenguaje de la labor de organización y sus consecuencias para los líderes», en Peter Drucker, El líder del futuro, Barcelona, Peter Drucker Fundation, DEUSTO.

Levitt, Peggy (2011), «A Transnational Gaze», Migraciones Internacionales, vol. 6, núm.1.

- y Nina Glick Schiller (2004), "Perspectivas internacionales sobre migración: conceptualizar la simultaneidad», Migración y Desarrollo, núm. 3. (2001), «The Transnational Villages», Berkeley and Los Angeles, University of California Press.

MASSEY, Douglas et al., (1990), Los ausentes: El proceso social de migración internacional en México Occidental, México, Alianza Editorial Mexicana, conaculta.

Meyer, J. y B. Rowan (1999), "Organizaciones institucionalizadas: la estructura formal como mito y ceremonia», en Paul DiMaggio y Wallter W. Powell (comps.), El nuevo institucionalismo en el análisis organizacional, México, Fondo de Cultura Económica.

MintzBerg, Henry (1995), La estructuración de las organizaciones, España, Ariel Economía.

Moctezuma Longoria, Miguel (2011), La transnacionalidad de los sujetos. Dimensiones, metodologías y prácticas convergentes de los migrantes mexicanos en Estados Unidos, Zacatecas, vaZ, Miguel Ángel Porrúa.

Montaño Hirose, Luis (2010), «Desempeño asociativo. El dilema de los modelos organizacionales», en Matilde Luna y Christina Puga (coords.), Nuevas perspectivas para el estudio de las asociaciones, México, UNAM, ANTROPOS.

$120 \frac{\text { PRIMER SEMESTRE } 2014}{\text { MIGRACIÓN Y DESARROLLO NÚM. } 22}$ 
(2001), «La razón, el afecto y la palabra: reflexiones en torno al sujeto en la organización», Iztapalapa, vol. 1, núm. 50.

MoYA, José (2005), «Immigrants and Associations: A Global and Historical Perspective», Journal of Ethnic and Migration Studies, vol. 31, núm. 5.

Orozco, Manuel y Katherine Welle (2005), «Hometown Associations and Development: Ownership, Correspondence, Sustainability, and Replicability», en Barbara J. Merz (comp.), New patterns for Mexico: observations on remittances, philanthropic giving, and equitable development, United States, Harvard University.

PapaIL, Jean y Jesús Arroyo Alejandre (2004), Los dólares de la migración, Zapopan, Universidad de Guadalajara, Institut De Recherche Pour Le Développment, Profmex, Casa Juan Pablos.

Pfefrer, Jeffery (1997), Nuevos rumbos en la teoría de la organización. Problemas y posibilidades, Oxford, University Press.

Ríos IBARRA, Ramón (2003), "Reapropiación de modelos y construcción de la identidad organizacional», Iztapalapa, vol. 2, núm.55.

Rivera-Salgado, Gaspar, Xóchitl Bada y Luis Escala Rabadán [conferencia] (2005), «Participación cívica y política de los migrantes mexicanos en Estados Unidos: las asociaciones de paisanos en Los Ángeles y Chicago», Washington DC, Woodrow Wilson International Center for Scholars, 4 y 5 de noviembre.

RombacH, Björn (1993), "Success at the expense of control», en Nils Brunsson y Johan P. Olsen (eds.), The Reforming Organization, New York, Routledge.

Scott, W. Richard (2005), "Organizaciones: Características duraderas y cambiantes», Gestión y Política Pública, vol. 14, núm. 3.

(2001), «Institutional Processes Affecting Organizational Structure and Perfomance», en Richard W. Scott, Institutions and Organizations, California, Foundations for Organizational Science.

y John W. Meyer (1999), «La organización de los sectores sociales: proposiciones y primeras evidencias», en Paul DiMaggio y Walter W. Powell (comps.), El nuevo institucionalismbo en el análisis organizacional, México, Fondo de Cultura Económica.

(1994), «Institutions and Organizations: Toward a Theoretical Synthesis», en W. Richard Scott y John W. Meyer (comps.), Institutional Environments and Organizations. Structural Complexity and Individualism, California, SAGE.

ZuCKer, Lynne G. (1999), «El papel de las instituciones en la persistencia cultural», en Paul DiMaggio y Walter W. Powell (comps.), El nuevo institucionalismo en el análisis organizacional, México, Fondo de Cultura Económica. 
Sitios de Internet consultados

Global Forum on Migration and Development (2013), «Unlocking the potential of migration for inclusive development», en http://gfmd_sweden2013-2014_ concept_paper(2).pdf, (08/01/2014).

Secretaría de Desarrollo Social (SEDESOL) (2010), "Reglas de operación Programa 3×1 para Migrantes», en http://normatecainterna.sedesol.gob.mx/disposiciones/ rop/rop_3x1_para_migrantes.pdf, (20/04/2014).

Secretaría de Relaciones Exteriores (SRE), [varios años], «Directorio de Organizaciones y Clubes de Oriundos», en http://www.ime.gob.mx/DirectorioOrganizaciones/, (26/04/2012).

Referencias de entrevistas

Presidente, Club Jiquilpan-USA (2012), por Carlos A. González Zepeda [trabajo de campo], Isomorfismo institucional y promoción del desarrollo local en asociaciones de migrantes michoacanos en Los Ángeles, California, California.

Vicepresidente, Club Jiquilpan- USA (2012), por Carlos A. González Zepeda [trabajo de campo], Isomorfismo institucional y promoción del desarrollo local en asociaciones de migrantes michoacanos en Los Ángeles, California, California.

Presidente, Club Los Reyes (2012), por Carlos A. González Zepeda [trabajo de campo], Isomorfismo institucional y promoción del desarrollo local en asociaciones de migrantes michoacanos en Los Ángeles, California, California.

Vicepresidente, Club Los Reyes (2012), por Carlos A. González Zepeda [trabajo de campo], Isomorfismo institucional y promoción del desarrollo local en asociaciones de migrantes michoacanos en Los Ángeles, California, California.

Presidente, Club Paisano Ciudad Hidalgo (2012), por Carlos A. González Zepeda [trabajo de campo], Isomorfismo institucional y promoción del desarrollo local en asociaciones de migrantes michoacanos en Los Ángeles, California, California.

Presidente, Club Huetamo (2012), por Carlos A. González Zepeda [trabajo de campo], Isomorfismo institucional y promoción del desarrollo local en asociaciones de migrantes michoacanos en Los Ángeles, California, California. 